\title{
INFLUÊNCIA DA ALTURA DE LEITO NO PROCESSO DE BIOSSORÇÃO DE NITRATO EM SEMENTES DE Moringa oleifera Lam. UTILIZANDO COLUNA DE LEITO FIXO
}

\author{
V. E. HORITA ${ }^{1}$, R. M. PAIXÃO ${ }^{1}$, I. M. RECK ${ }^{2}$ e A. M. S. VIEIRA ${ }^{2}$ \\ ${ }^{1}$ Universidade Estadual de Maringá, Departamento de Engenharia Química \\ ${ }^{2}$ Universidade Estadual de Maringá, Departamento de Engenharia de Alimentos \\ E-mail para contato: ellen909@ hotmail.com
}

\begin{abstract}
RESUMO - Existe certa concordância global que os teores de nitrato em sistemas hídricos não devem atingir valores acima do limite máximo de potabilidade vigente estabelecido pela Portaria MS. $\mathrm{n}^{\circ} 2.914 / 2011$ de $45 \mathrm{mg} \mathrm{L}^{-1} \mathrm{NO}_{3}{ }^{-}$, devido aos riscos comprovados de danos à saúde pública. Assim, é imprescindível a remoção deste poluente presente em água; uma das técnicas promissoras devido a sua elevada eficiência é a adsorção desse íon por biossorventes. Dessa forma, estudou-se a biossorção de nitrato em coluna leito fixo com sementes de Moringa oleifera Lam., e os ensaios referentes à influência da altura de leito demonstraram que este parâmetro influencia no processo de biossorção, sendo que uma maior altura de leito promove elevados valores de capacidade de adsorção $\left(\mathrm{q}_{\mathrm{t}}\right)$.
\end{abstract}

\section{INTRODUÇÃO}

Uma vez que a contaminação de águas subterrâneas e superficiais por nitrato limita o seu uso como recurso hídrico, por este ser uma ameaça à saúde humana em valores superiores a $45 \mathrm{mg} \mathrm{L}^{-1}$ de $\mathrm{NO}_{3}^{-}$, teor estabelecido pela Portaria no 2.914/2011, do Ministério da Saúde, em concordância aos limites avaliados pela Agência de Proteção Ambiental dos Estados Unidos (Doudrick et al., 2013), é pertinente seu tratamento visando a potabilidade. Dentre as técnicas de tratamento existentes para remoção deste íon, a adsorção parece ser a técnica mais atrativa, em termos de custo, simplicidade de design e de operação, e capacidade regenerativa, além de reduzir/minimizar diferentes tipos de poluentes de água e efluentes (Bhatnagar e Sillanpaa, 2011; Jain et al., 2015).

O processo de adsorção em leito fixo consiste em um fenômeno contínuo e prático para realização de ciclos de adsorção e dessorção, em comparação ao método de batelada, pois faz um melhor uso do gradiente de concentração ao movimentar as moléculas de adsorbato transpondo os sítios ativos de forma proporcional ao longo da coluna e acentuando a força motriz da operação. Além disso, permite uma utilização mais eficiente da capacidade do adsorvente, resultando em uma melhor qualidade do efluente em condições ideais, por tratar um considerável volume de água residual por meio de uma quantidade relativamente pequena de adsorvente empacotado na coluna (Aksu e Gönen, 2004).

Com isso, é essencial que o processo apresente alta eficiência associada a baixo custo, o que pode ser conferido pelo uso de adsorventes naturais como a Moringa oleifera Lam. 
(Formentini-Schmitt et al., 2013). A Moringa oleifera Lam. é uma planta originária do nordeste da Índia, de próspero cultivo em clima tropical portanto amplamente aplicável nacionalmente, e reconhecida por seus atributos nutricionais, medicinais e de limpeza da água (Akhtar et al.,2007) sendo utilizada principalmente em processos de coagulação, clarificação e adsorção em soluções aquosas na remoção de poluentes (Meneghel et al., 2013).

Em conformidade ao exposto, o presente trabalho objetivou investigar a influência da variável altura de leito na quantidade de nitrato adsorvida em ensaios de biossorção em coluna de leito fixo empacotada com sementes de Moringa oleifera Lam.

\section{MATERIAIS E MÉTODOS}

\subsection{Preparo e Análise das Soluções}

Foram preparadas soluções sintéticas de nitrato a partir da dissolução de nitrato de sódio $\left(\mathrm{NaNO}_{3}\right)$ em água deionizada. As concentrações iniciais e finais foram determinadas por Espectrofotometria UV-VIS (HACH DR 5000) no comprimento de onda de $205 \mathrm{~nm}$ conforme proposto por Instituto Adolfo Lutz (2004).

\subsection{Preparo do Material Biossorvente}

As sementes de Moringa oleifera Lam foram descascadas, secas em estufa com circulação de ar à $40^{\circ} \mathrm{C}$ até peso constante. Em seguida, foram trituradas e homogeneizadas em um processador e peneiradas em $0,8 \mathrm{~mm}$, em peneiras série agitador tipo magnético (Bertel).

\subsection{Ensaios de Biossorção em coluna de leito fixo}

Os ensaios de biossorção em coluna de leito fixo foram conduzidos em uma coluna de vidro de $28 \mathrm{~cm}$ de altura e $0,9 \mathrm{~cm}$ de diâmetro interno, posicionada verticalmente, de forma que o biossorvente foi empacotado com base na metodologia "slurry method" descrita por Zach-Maor et al. (2011). Assim, 1/4 da coluna foi preenchida com esferas de vidro, seguida de um pequeno pedaço de tela de poliamida, de modo que o biossorvente não ficasse em contato com as esferas de vidro, seguida das sementes de Moringa oleifera Lam. na granulometria desejada e umedecidas com água deionizada, por fim, adicionou-se outro pedaço pequeno de tela de poliamida e mais $1 / 4$ da altura da coluna foi completado com esferas de vidro, para garantir que a altura do leito não fosse alterada durante o ensaio.

As soluções aquosas de nitrato, na concentração inicial de $100 \mathrm{mg} \mathrm{L}^{-1}$ e pH sem ajuste $(5,5)$, foram alimentadas ao leito na vazão de $1 \mathrm{~mL} \min ^{-1}$, em fluxo ascendente, por uma bomba peristáltica Masterflex, e os ensaios foram conduzidos utilizando altura do leito de 2,5 $\mathrm{cm}, 5,0 \mathrm{~cm}$ e 7,5 cm, que correspondem respectivamente as massas de 1,2 e 3 gramas de sementes de Moringa oleifera Lam. empacotadas em temperatura ambiente $\left(25{ }^{\circ} \mathrm{C}\right)$. As amostras do efluente foram coletadas em intervalos de tempo pré-estabelecidos, até atingir a saturação do leito em $\mathrm{C}_{\mathrm{t}} / \mathrm{C}_{0}=0,9$.

A capacidade de adsorção do leito pode ser obtida pelo cálculo da área abaixo da curva de ruptura, dada pela Equação 1: 


$$
q_{t}=\frac{Q A}{1000}=\frac{Q}{1000} \int_{t=0}^{t=t_{\text {tatal }}} C_{\text {ad }} d t
$$

Em que $\mathrm{q}_{\mathrm{t}}$ é a quantidade total de soluto adsorvida no leito em $\mathrm{mg}$; $\mathrm{t}_{\text {total }}$ é o tempo total de alimentação expresso em min; Q é a vazão de alimentação em $\mathrm{mL} \mathrm{min}^{-1}$; A é a área abaixo da curva de ruptura e $\mathrm{C}_{\mathrm{ad}}$ é a concentração adsorvida no leito em $\mathrm{mg} \mathrm{L}^{-1}$.

O termo " $\mathrm{C}_{\mathrm{ad}}$ " apresentado na Equação 1, pode ser obtido por meio do cálculo da diferença entre a concentração de alimentação do leito e a concentração do efluente, dado pela Equação 2:

$$
C_{\text {ad }}=C_{0}-C_{t}
$$

Em que $C_{a d}$ é a concentração adsorvida no leito em $\mathrm{mg} \mathrm{L}^{-1}$ e $\mathrm{C}_{0}$ e $\mathrm{C}_{\mathrm{t}}$ são as concentrações inicial e final em $\mathrm{mg} \mathrm{L}^{-1}$.

A capacidade máxima de adsorção do leito $\mathrm{q}_{\mathrm{e}}$, em $\mathrm{mg} \mathrm{g}^{-1}$, indica a quantidade total de soluto adsorvido $\left(\mathrm{q}_{\mathrm{t}}\right)$ por unidade de massa de adsorvente $\mathrm{m}$ ao final do tempo total de alimentação.

$$
q_{e}=\frac{q_{t}}{m}
$$

A quantidade total de adsorbato recebida pelo leito ao final do tempo total de alimentação $\mathrm{W}_{\mathrm{t}}$, pode ser calculada utilizando a Equação 4 .

$$
W_{t}=\frac{C_{0} Q t_{s}}{1000}
$$

Em que $\mathrm{C}_{0}$ é a concentração de alimentação em $\mathrm{mg} \mathrm{L}^{-1}$, Q é a vazão de alimentação em $\mathrm{mL} \min ^{-1}$ e $t_{\mathrm{s}}$ é o tempo necessário para o leito atingir a saturação expresso em min.

\subsection{Modelagem das Curvas de Ruptura: O Modelo de Thomas}

O modelo de Thomas é regularmente usado na modelagem matemática do processo de adsorção em sistemas contínuos, como em leito fixo, para analisar os dados de colunas em escala de laboratório e fornecer uma previsão da curva de ruptura (Meng et al., 2013). A Equação 5 representa a forma não-linear do modelo de Thomas:

$$
\frac{C_{t}}{C_{0}}=\frac{1}{1+e^{\left(\frac{k_{T H}}{Q}\right)\left(q_{0} m-C_{0} V_{e f}\right)}}
$$

Em que $\mathrm{C}_{0}$ e $\mathrm{C}_{\mathrm{t}}$ são as concentrações de alimentação e do efluente em mg $\mathrm{L}^{-1}$, $\mathrm{k}_{\mathrm{TH}}$ é o coeficiente da transferência de massa em $\mathrm{mL} \mathrm{mg}^{-1} \mathrm{~min}^{-1}$, $\mathrm{q}_{0}$ é a capacidade de adsorção do leito em $\mathrm{mg} \mathrm{g}^{-1}, \mathrm{~m}$ é a massa de adsorvente em $\mathrm{g}$, Q é a vazão de alimentação em $\mathrm{mL} \mathrm{min}^{-1} \mathrm{e}$ $\mathrm{V}_{\text {ef }}$ é o volume de efluente enviado à coluna em $\mathrm{L}$. 


\section{RESULTADOS E DISCUSSÃO}

\subsection{Ensaios de biossorção em coluna de leito fixo}

As curvas de ruptura construídas a fim de avaliar a influência do parâmetro altura de leito na biossorção de nitrato estão demonstrados na Figura 1.

Figura 1 - Curvas de ruptura obtidas na avaliação do efeito da altura de leito na biossorção de nitrato em sementes de Moringa oleifera Lam.

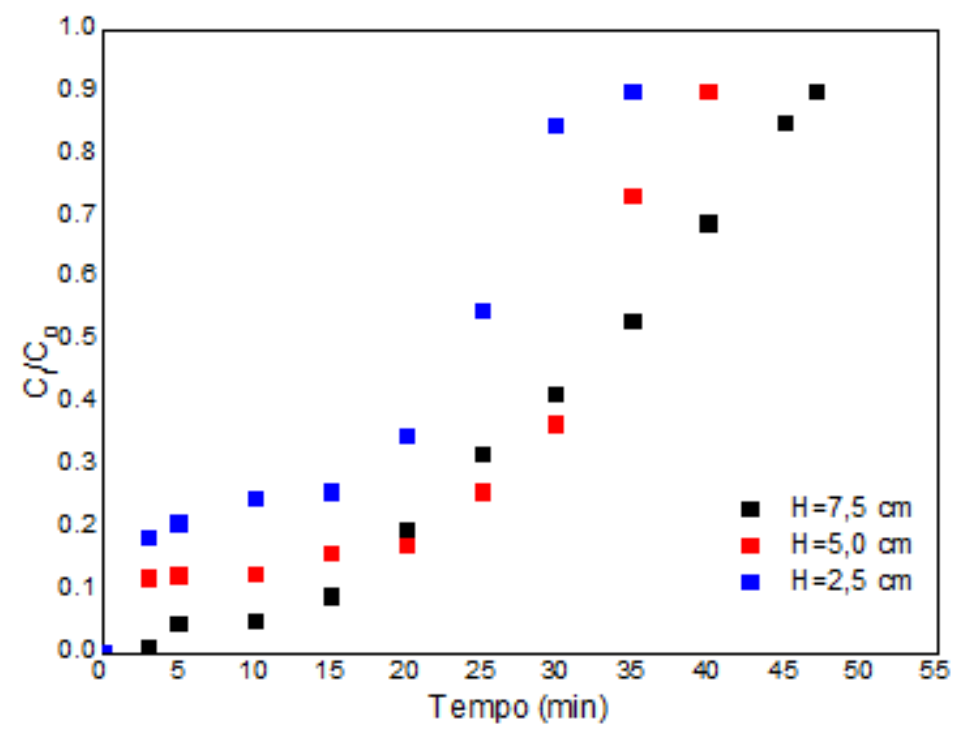

Pode-se observar na Figura 1 que com o aumento da altura de leito (massa de biossorvente) há um aumento no tempo de saturação, que passou de 33 minutos na altura de $2,5 \mathrm{~cm}$ para 47 minutos na altura de $7,5 \mathrm{~cm}$. Comportamento semelhante foi reportado por $\mathrm{Xu}$ et al. (2013), o que indica que à medida que a solução de nitrato flui para a coluna, o ponto na curva "em forma de S" se aproxima do seu valor de exaustão, de forma que o tempo de ruptura cresce com o aumento da altura do leito.

Os valores calculados para a quantidade total de soluto adsorvida no leito $\left(\mathrm{q}_{\mathrm{t}}\right)$, capacidade máxima de adsorção do leito $\left(\mathrm{q}_{\mathrm{e}}\right)$ e quantidade total de adsorbato enviada ao leito ao final do tempo total de alimentação $\left(\mathrm{W}_{\mathrm{t}}\right)$ encontram-se na Tabela 1. De acordo com os valores obtidos, observa-se que o valor de $\mathrm{q}_{\mathrm{t}}$ aumenta com a maior altura de leito, devido ao aumento nos sítios de biossorção disponíveis, pela maior quantidade de biossorvente no leito. Esse mesmo comportamento foi reportado por Golie e Upadhyayula (2016) também na adsorção de nitrato em coluna de leito fixo, utilizando composto de quitosana/alumina.

Tabela 1 - resultados obtidos na análise do efeito da altura de leito

\begin{tabular}{|c|c|c|c|}
\hline $\begin{array}{c}\text { Altura do } \\
\text { leito }(\mathrm{cm})\end{array}$ & $\mathrm{q}_{\mathrm{t}}(\mathrm{mg})$ & $\mathrm{q}_{\mathrm{e}}\left(\mathrm{mg} \mathrm{g}^{-1}\right)$ & $\mathrm{W}_{\mathrm{t}}(\mathrm{mg})$ \\
\hline \hline 2,5 & 2,011 & 2,011 & 3,341 \\
\hline 5,0 & 2,753 & 1,376 & 3,919 \\
\hline 7,5 & 3,094 & 1,031 & 4,711 \\
\hline
\end{tabular}




\subsection{Ensaios de biossorção em coluna de leito fixo}

O modelo de Thomas foi ajustado aos dados experimentais obtidos neste estudo, utilizando o software Origin Pro® versão 9.0, e as curvas obtidas por meio do ajuste estão apresentadas na Figura 2 e seus respectivos parâmetros na Tabela 2.

Figura 2 - Modelagem das curvas de ruptura utilizando o Modelo de Thomas.

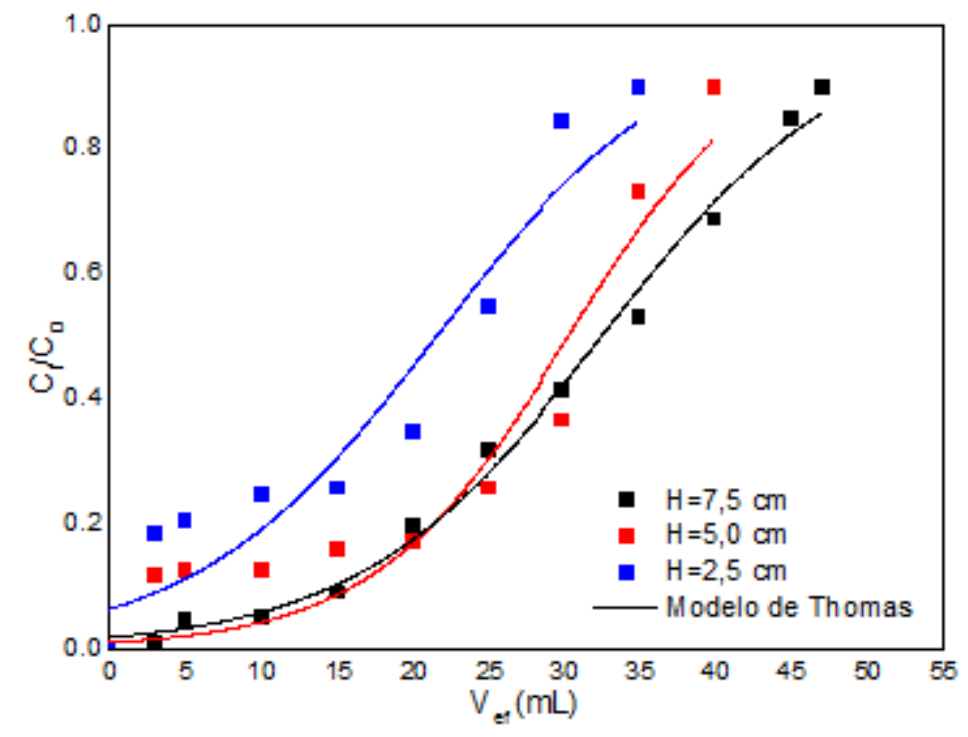

Tabela 2 - Parâmetros obtidos a partir do ajuste do Modelo de Thomas aos dados experimentais

\begin{tabular}{|c|c|c|c|}
\hline Altura do leito $(\mathrm{cm})$ & 2,5 & 5,0 & 7,5 \\
\hline \hline $\mathrm{q}_{0}\left(\mathrm{mg} \mathrm{g}^{-1}\right)$ & 2,156 & 1,518 & 1,085 \\
\hline $\mathrm{k}_{\mathrm{TH}}\left(\mathrm{mL} \mathrm{mg}^{-1} \mathrm{~min}^{-1}\right)$ & 1,256 & 1,541 & 1,238 \\
\hline $\mathrm{R}^{2}$ & 0,9164 & 0,9096 & 0,9928 \\
\hline
\end{tabular}

Por meio da Tabela 2 pode-se verificar que o modelo de Thomas apresentou bom ajuste aos dados experimentais obtidos a partir da análise da influência da altura de leito na biossorção de nitrato em sementes de Moringa oleifera Lam. em processo de coluna em leito fixo, com correlações superiores a 90\%. Além disso, o parâmetro $\mathrm{q}_{0}$ se assemelha ao encontrado experimentalmente $\left(\mathrm{q}_{\mathrm{e}}\right)$, o qual foi reduzido com o aumento da altura de leito.

\section{CONCLUSÃO}

A influência da altura de leito na biossorção de nitrato em coluna de leito fixo utilizando sementes de Moringa oleifera Lam. como biossorvete foi estudada e os resultados sugerem que o aumento da altura do leito (massa de biossorvente) acarreta em maior quantidade total de soluto adsorvida no leito.

O modelo de Thomas foi utilizado para descrever o comportamento das curvas de ruptura obtidas experimentalmente, e reproduziu bem os dados, com correlações superiores a $90 \%$ para as três alturas estudadas. 


\section{REFERÊNCIAS}

AKSU, Z.; GÖNEN, F. Biosorption of phenol by immobilized activated sludge in a continuous packed bed: prediction of breakthrough curves. Process Biochem., v. 39, n. 5, p. 599-613, 2004.

AKHTAR, M.; HASANY, S. M.; BHANGER, M. I., IQBAL, S. Sorption potential of Moringa oleifera pods for the removal of organic pollutants from aqueous solutions. $J$. Hazard. Mater., v. 141, n. 3, p. 546-556, 2007.

BHATNAGAR, A.; SILLANPAA, M. A review of emerging adsorbents for nitrate removal from water. Chem. En. J., v.168, p.493-504, 2011.

BRASIL, Portaria nº 2914 de 12 de Dezembro de 2011, Ministério da Saúde, 2011.

DOUDRICK, K.; YANG, T.; HRISTOVSKI, K.; WESTERHOFF, P. Photocatalytic nitrate reduction in water: Managing the hole scavenger and reaction by-product selectivity. Applied Catalysis B: Environmental, p. 136- 137, 2013.

FORMENTINI-SCHMITT, D. M.; ALVES, A. C. D.; VEIT, M. T.; BERGAMASCO, R.; VIEIRA, A. M. S.; FAGUNDES-KLEN, M. R. Ultrafiltration Combined with Coagulation/Flocculation/Sedimentation Using Moringa oleifera as Coagulant to Treat Dairy Industry Wastewater. Water Air Soil Pollut, 224: 1682, 2013.

GOLIE, W. M.; UPADHYAYULA, S. Continuous fixed-bed column study for the removal of nitrate from water using chitosan/alumina composite. J. of Water Process En.,, v.12, p.58-65, 2016.

INSTITUTO ADOLFO LUTZ. Métodos físico-químicos para análise de alimentos. 2008.

JAIN, S.; BANSIWAL, A.; BINIWALE, R. B.; MILMILLE, S.; DAS, S.; TIWARI, S.; ANTONV, P. S. Enhancing adsorption of nitrate using metal impregnated alumina. $J$. Environmental Chem. En., v.3, p.2342-2349, 2015.

MENEGHEL, A. P.; GONÇALVES Jr., A. C.; RUBIO, F.; DRAGUNSKI, D. C.; LINDINO, C. A.; STREY, L. Biosorption of Cadmium from water using Moringa (Moringa oleifera Lam.) Seeds. Water Air Soil Pollut., v.224, p.1383-1396, 2013.

MENG, M.; FENG, Y.; ZHANG, M.; LIU Y.; JI, Y.; WANG, J; WU, Y.; YAN, Y. Highly efficient adsorption of salicylic acid from aqueous solution by wollastonite-based imprinted adsorbent: A fixed-bed column study. Chem. En. J., v. 225, p. 331-339, 2013.

XU, X.;GAO, B.; TAN, X.; ZHANG, X.; YUE, Q.; WANG, Y.; LI, Q. Nitrate adsorption by stratified wheat resin in lab-scale columns. Chem. En. J., v.226, p.1-6, 2013.

ZACH-MAOR, A.; SEMIAT, R.; SHEMER, H. Fixed bed phosphate adsorption by immobilized nano-magnetite matrix: experimental and a new modeling approach. Adsorption, v. 17, n. 6, p.929-936, 2011. 